\title{
Medicina narrativa: la comunicazione farmacista-paziente migliora l'adesione alla terapia?
}

\author{
Sabrina Cherchi ${ }^{1}$, Sara Sanna ${ }^{2}$, Francesco Logias ${ }^{3}$, Pasquale Sanna ${ }^{1}$, Alessandro Carrus ${ }^{3}$, Simona Sanna ${ }^{4}$ \\ ${ }^{1}$ U.O.C. Servizio Farmaceutico Territoriale, ATS. Sardegna, ASSL Nuoro, Nuoro \\ ${ }^{1}$ U.O.C. Farmacia Ospedaliera, P.O. San Francesco, ATS. Sardegna, ASSL Nuoro, Nuoro \\ ${ }^{3}$ U.O.C. Nefrologia e Dialisi, P.O. San Francesco, ATS. Sardegna, ASSL Nuoro, Nuoro \\ ${ }^{4}$ Libero Professionista, Nuoro
}

\begin{abstract}
Narrative medicine: does pharmacist-patient communication improve medication adherence?
In Western countries, medication adherence reaches only about $50 \%$ among patients affected by chronic diseases who require complex and long-term therapies. The lack of adherence causes suboptimal clinical results and increases National Healthcare System (NHS) spending. Considering the progressive aging of the general population, the increase in chronic diseases and the decrease in resources, enhancement of adherence will improve the allocation of resources as well as patients' quality of life. An overview of the literature suggests that improving therapy adherence may improve results more effectively than other therapeutic interventions. The research project described in this report focused on the communication between patient and pharmacist and aimed to evaluate the feedback on therapy adherence in hemodialysis patients. Narrative medicine and multivariate statistical analysis were employed and compared. Structured interviews were conducted with hemodialysis patients treated at the Nephrology and Dialysis Unit of the San Francesco Hospital in Nuoro, Sardinia. The results suggested that the alliance with the pharmacist could be greatly improved in relevant aspects, including paying more attention to the patient's psychophysical conditions. Pharmacological counseling may thus allow to improve therapy adherence. The narrative interview revealed the need of patients to communicate more and to form an alliance with the pharmacist that is crucial to enhancing adherence. This experience may support the practical use of narrative medicine in identifying patients' needs and suggesting corrections to current practice.
\end{abstract}

Keywords: Clinical pharmacist, Medication adherence, Narrative medicine, Pharmacist-patient relationship

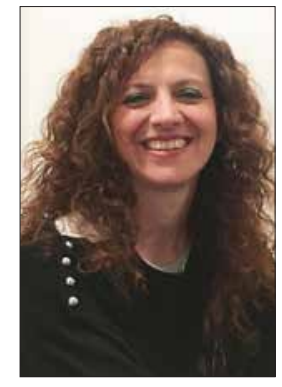

Introduzione

L’aderenza al trattamento è generalmente definita come la misura in cui il paziente assume il farmaco prescritto agli intervalli e ai dosaggi indicati dal medico prescrittore. Un aspetto importante, oltre al ruolo attivo del paziente e alla sua partecipazione al trattamento, è che essa si riferisce generalmente

Sara Sanna

Accepted: April 28, 2017

Published online: June 6, 2017

Indirizzo per la corrispondenza:

Dr.ssa Sara Sanna

Farmacia Ospedaliera, ATS Sardegna, ASSL Nuoro

Via Mannironi

08100 Nuoro

sarasanna975@gmail.com ai consigli ricevuti da tutti i professionisti sanitari. Possiamo introdurre a tal proposito il termine concordanza, che non implica alcun aspetto riguardante l'assunzione del farmaco, ma che si riferisce al grado di alleanza terapeutica stabilita tra professionista sanitario e paziente e che, peraltro, assume una valenza più ampia (1).

A differenza della compliance, dove il paziente segue passivamente le indicazioni del medico, l'aderenza presuppone un'alleanza terapeutica; secondo Edward Bordin (2), questa è costituita da tre componenti: l'esplicita condivisione di obiettivi da parte del paziente e del terapeuta, la chiara definizione di compiti reciproci all'inizio del trattamento e il tipo di legame affettivo che si costituisce tra i due, caratterizzato da fiducia e rispetto.

L'aderenza del paziente al trattamento prescritto dal medico è un processo comportamentale complesso e può essere influenzato da molteplici fattori, raggruppati, secondo l'Organizzazione Mondiale della Sanità (OMS), in 5 classi: fattori socioeconomici, fattori correlati al paziente (dimenticanza, negazione della malattia, credo religioso, scarsa comprensione), fattori correlati alla condizione clinica (sintomi non 
evidenti o molto lievi o addirittura molto severi, stato confusionale), fattori correlati al Sistema Sanitario e al team degli operatori sanitari e fattori correlati alla terapia (preparazione difficoltosa, regime complesso, frequenza elevata di assunzione, effetti indesiderati e reazioni avverse ai farmaci) (3) .

Diversi studi hanno dimostrato che vari fattori alla base della mancata aderenza intenzionale possono svolgere un ruolo anche nella mancata aderenza non intenzionale. Per esempio, i pazienti che temono effetti dannosi hanno maggiori probabilità di seguire meno coscienziosamente o di dimenticare l'assunzione della terapia e, talvolta, di ometterla volontariamente (4).

Tale condizione produce una riduzione del beneficio o un aumento del rischio associato alla terapia, alterando significativamente il rapporto rischio/beneficio; le conseguenze possono essere estremamente gravi, mettendo talvolta a rischio la sopravvivenza. Spesso l'aderenza terapeutica è influenzata da una comunicazione non accurata o incompleta tra gli operatori sanitari e i pazienti, e questo rappresenta uno dei fattori determinanti per il verificarsi di eventi avversi (5).

Strumenti pratici e concettuali per migliorare l'aderenza terapeutica e comprendere il paziente vengono forniti dalla Medicina Narrativa, con cui si intende una metodologia di intervento clinico-assistenziale basata su una specifica competenza comunicativa (6). Tale metodo integra la medicina basata sulle prove di efficacia con le esperienze e le aspettative del paziente per favorire un percorso di cura condiviso. Il paziente assume un ruolo di protagonista nella storia della propria malattia, pertanto attraverso la narrazione è possibile sviluppare un percorso personalizzato, appropriato e in linea con le indicazioni dell'Evidence based medicine (EBM). Non vi è una contrapposizione tra la medicina narrative-based (NBM) e l'EBM, ma, al contrario, vi è un'integrazione eccellente; la prima fortifica la seconda rendendola più completa. Raccontare la propria storia di malattia nella NBM non è meno rilevante dell'esposizione dei sintomi clinici.

Attraverso I'utilizzo di questo modello, sviluppato presso la Harvad Medical School da B.J. Good (7), si evince l'importanza dei "racconti" nel valutare la qualità delle cure e del rapporto medico-infermiere-farmacista/paziente; questo perché le narrazioni di malattia sono un ottimo strumento di comprensione della relazione del paziente con la malattia stessa.

Uno degli obiettivi primari della medicina narrativa è quello di migliorare l'adesione terapeutica e la partecipazione del paziente alla sua malattia, consolidando il rapporto con gli operatori sanitari; è necessario fare questo poiché ogni vera relazione, anche quella terapeutica, richiede un dialogo. Spesso in medicina il paziente è definito caso clinico e la patologia diventa la sola e unica protagonista ponendo il malato in secondo piano (8).

Con la medicina narrativa si cerca di facilitare la partecipazione attiva dei pazienti e di migliorare il funzionamento dell'intero gruppo di cura; l'ascolto favorisce la fiducia e au- menta l'alleanza terapeutica.

In questo quadro di figure associate, il farmacista riveste un ruolo fondamentale nel migliorare l'aderenza alla terapia, essendo l'esperto del farmaco e di tutte le problematiche ad esso connesse; può contribuire efficacemente alla riduzione della mancata aderenza terapeutica nei pazienti cronici in particolar modo, a supporto dell'attività del medico $(9,10)$. Il paziente deve avere un confronto attivo e una buona comunicazione con il personale sanitario; è dunque importante essere in grado di ascoltare e rispondere alla persona, non solo al sintomo; parliamo quindi di "Farmacia Narrativa" (11) ovvero la medicina narrativa applicata alla farmacia come strumento di riscoperta dell'identità professionale, del rapporto farmacista-paziente.

A tal proposito si parla spesso di counseling, termine inglese che deriva dal verbo to counsel (dare consigli); secondo Rollo May e Rogers (padri fondatori del counseling), il counselor ha il compito di favorire lo sviluppo e le potenzialità del "cliente", aiutandolo a superare eventuali problemi di personalità che gli impediscono di esprimersi. Pertanto il counselor rappresenta una guida (12) per il paziente, pensiero condiviso anche dall'OMS, riconoscendo nel counseling farmacologico un processo che, attraverso il dialogo e l'interazione, aiuta i pazienti a gestire i propri problemi e a prendere decisioni. In particolar modo il couseling per il farmacista consente di integrare la consulenza tecnico-scientifica con l'interesse vero e proprio nei confronti del paziente, ascoltando la sua storia e la sua esperienza personale; si costruisce così un rapporto di fiducia con un conseguente miglioramento dell'aderenza terapeutica $(13,14)$.

Per avere un riscontro su quanto scritto e pertanto verificare se in seguito all'instaurarsi di una relazione farmacista-paziente migliora l'adesione terapeutica, è stato condotto uno studio nell'ospedale San Francesco di Nuoro, U.O. di Nefrologia e Dialisi. L'obiettivo della ricerca è quello di comprendere, attraverso le narrazioni dei pazienti, il loro pensiero riguardo alla figura del farmacista e all'inserimento di questo all'interno dell'equipe sanitaria che segue il loro percorso, individuare i punti di forza e di debolezza del rapporto farmacista-paziente per accrescere il valore e il ruolo del farmacista ospedaliero e cogliere l'importanza della narrazione e del dialogo per il paziente.

\section{Metodi}

Lo studio è stato realizzato mediante somministrazione di interviste guidate secondo il questionario riportato in Appendice 1. Lo studio ha inizialmente riguardato un campione di oltre 90 pazienti. Su questo numero è stata poi effettuata un'attenta valutazione qualitativa al fine di eliminare i questionari non completi o quelli caratterizzati da una certa "incoerenza" nelle risposte. Per esempio, alcuni pazienti hanno dichiarato di non avere alcun rapporto con il farmacista poiché delegano completamente a "terzi" qualsiasi relazione con lo stesso in ambito di ritiro e/o richiesta di chiarimenti del 
farmaco. Al termine di questa attenta fase selettiva di tipo qualitativo, i questionari ritenuti "validi" e completamente aderenti all'obiettivo dello studio sono 60 .

Il questionario è stato suddiviso in varie parti attinenti alle caratteristiche anagrafiche, sociali e culturali degli intervistati, alla gestione della terapia a domicilio e al rapporto esistente o che vorrebbero avere con il farmacista.

Il questionario è stato dunque strutturato in maniera tale da inquadrare nel migliore dei modi, dal punto di vista statistico, le principali caratteristiche della popolazione indagata così da comprendere quali fossero i fattori che influenzano maggiormente le successive risposte sui farmaci e sul rapporto con il farmacista.

Tutti i questionari sono stati compilati seguendo un rigido protocollo volto a garantire e a soddisfare il pieno anonimato del paziente. Il protocollo prevedeva che solo una persona tra gli autori della presente ricerca potesse condurre le interviste e che durante il loro svolgimento non fossero presenti altre persone nello studio. Con la sola eccezione del supporto cartaceo, non è mai stato registrato il contenuto delle interviste sotto alcuna forma temporanea e/o permanente né audio/ video. Una volta compilati, i questionari sono stati archiviati e identificati mediante un apposito codice alfanumerico, in modo da evitare qualsiasi tipo di associazione diretta/indiretta con il paziente. Le risposte sono state inserite in un apposito database, utilizzando una codifica numerica di tipo quantitativo (Appendice 1).

Nell'ultima parte del questionario sono state predisposte tre domande a risposta aperta, in modo da permettere al paziente di dialogare, raccontare e, per l'appunto, "narrare". È stata svolta un'analisi qualitativa, utilizzando lo strumento della narrazione, consentendo di sviluppare una riflessione rispetto alla modalità espressiva e relazionale nel percorso assistenziale del paziente, ma anche sugli atteggiamenti del personale sanitario, favorendo il miglioramento dell'attenzione alla storia personale delle persone assistite.

Le analisi statistiche sono state condotte prevalentemente mediante l'ausilio del software Statistica ${ }^{\circledR} 7.1$ (15) e il programma open source $R(16)$. Sono state effettuate differenti elaborazioni statistiche a partire dalle più semplici tecniche di analisi statistica monovariata (media, mediana, minimo, massimo, errore standard) alle più complesse elaborazioni di statistica bi- e multivariata.

L'analisi statistica bivariata è stata condotta mediante matrice di correlazione di Pearson per stimare il grado di correlazione tra i parametri indagati. II livello di significatività è stato posto pari a $\mathrm{p}<0.05$. Il coefficiente di correlazione di Pearson è una misura di relazione tra due variabili e determina fino a che livello le due variabili risultano tra loro "proporzionali", cioè relazionate linearmente (17).

Sono poi state condotte le analisi dei fattori principali (Principal Factor Analysis, PFA). È un metodo di analisi multivariata volto a spiegare le correlazioni fra un insieme di $p$ variabili osservate attraverso un insieme di $n$ variabili non osser- vate (latenti) a cui si dà il nome di "fattori" (18). Per facilitare l'interpretazione dei risultati, è stata utilizzata la rotazione varimax, poiché è una rotazione ortogonale che cerca di modificare i pesi fattoriali in modo da massimizzare la varianza fra i pesi fattoriali relativi ad ogni fattore, semplificando l'interpretazione. I valori riportati attengono alla mediaterrore standard.

\section{Risultati}

La popolazione indagata si caratterizza per una prevalenza del genere maschile (75\%). Dal punto di vista anagrafico si denota una netta preponderanza di persone con età maggiore ai 71 anni (43\%) e una percentuale estremamente bassa di pazienti con età inferiore ai 40 anni (2\%); l'età media si attesta sui $63 \pm 2$ anni. Questi dati risultano coerenti con i seguenti due aspetti fondamentali in termini di incidenza della patologia (19): 1) maggiore nel genere maschile e 2) notoriamente più elevata nella popolazione anziana.

La popolazione indagata risulta residente nella sola provincia di Nuoro, che è anche il luogo di nascita di buona parte degli intervistati, fattore questo legato al fatto che lo studio è stato svolto nei centri dialisi afferenti alla U.O. di Nefrologia e Dialisi del Presidio Ospedaliero di Nuoro.

Le persone intervistate risultano nel $48 \%$ dei casi rilevati sposate o conviventi, nel $30 \%$ dei casi single, nel $15 \%$ dei casi separate e nel $7 \%$ dei casi vedove. In generale, il tasso di scolarizzazione degli intervistati risulta abbastanza basso, infatti il 64\% di essi dichiara di non avere alcun titolo di studio oppure di aver completato il ciclo di scuola elementare (13\%). Solamente il $23 \%$ è in possesso di un diploma superiore e nessuno degli intervistati dichiara di possedere la laurea o un titolo superiore.

La matrice di correlazione (MC) di Pearson evidenzia diverse interessanti correlazioni altamente significative (Tab. I). Di seguito si discutono le più interessanti.

Si evidenziano subito alcuni rilevanti "fattori sociali" tra cui una correlazione positiva tra età e status di famiglia $(R=$ $0.39 ; p<0.05)$, che indica come nella popolazione più adulta aumentino i coniugati, e una correlazione negativa con il titolo di studio ( $R=-0.28 ; p<0.05)$, a significare che la popolazione più adulta è anche quella che si caratterizza per i più bassi tassi di scolarizzazione.

La matrice mostra come all'aumentare dell'età i pazienti tendano a delegare una terza persona al ritiro dei farmaci $(R=-0.37 ; p<0.05)$ e, non gestendo più in autonomia la propria terapia $(R=-0.34, p<0.05)$, si affidino prevalentemente sia per l'acquisto $(R=-0.33 ; p<0.05)$ che per la somministrazione $(R=-0.34 ; p<0.05)$ a parenti stretti, dal coniuge passando per i figli e i fratelli/sorelle $(R=0.33 / 0.36 p<0.05)$. Osservando gli aspetti più prettamente connessi al rapporto con il farmacista, si denota come l'età non influisca in alcun modo (né positivo né negativo) nel rapporto con questo.

$\mathrm{Vi}$ sono due correlazioni attinenti al genere. Nel primo 
caso si nota come la popolazione maschile si senta poco aiutata dal farmacista in caso di difficoltà, il che vuol dire che è vero l'opposto per quella femminile $(R=-0.31 ; p<0.05)$. Un'ulteriore evidenza è che il genere maschile ritiene poco rilevante l'aspetto psicologico tra le considerazioni di cui il farmacista dovrebbe tenere conto durante il ritiro del farmaco. Anche in questo caso è vero l'opposto per il genere femminile, che sembra invece considerare simili aspetti abbastanza importanti $(R=-0.31 ; p<0.05)$.

Anche lo status di famiglia dei pazienti mostra alcune interessanti correlazioni. La MC mostra come nei pazienti caratterizzati da più stretti legami parentali (per esempio, sposati e con figli) il ritiro dei farmaci venga spesso "delegato" $(R=-0.27$; $p<0.05)$ ai parenti più stretti $(R=0.40 ; p<0.05)$; è interessante notare come la stessa cosa valga anche per l'assunzione del farmaco, che viene spesso somministrato da un familiare stretto.

Per quanto attiene al titolo di studio si rileva come siano soprattutto i pazienti dotati di un grado d'istruzione più elevato a prediligere il ritiro diretto del farmaco senza delega a "terzi". Inoltre, questa tipologia di pazienti rileva come il farmacista dia solitamente poco peso allo stato psicologico del paziente quando ritira il farmaco $(R=-0.27 ; p<0.05)$. Questo sembra indicare come questi pazienti gradirebbero una maggiore attenzione del farmacista su tali aspetti.

Dai risultati della MC non si rilevano invece correlazioni statisticamente significative con il parametro "anni di trattamento".

Per quanto riguarda la conservazione del farmaco la MC rileva come in coloro che delegano la somministrazione a "terzi" la stessa avvenga prevalentemente utilizzando un medicinale conservato nei portapillole. Coloro che conservano il farmaco prevalentemente nel portapillole tendono anche a seguire poco le istruzioni ( $R=-0.35 ; p<0.05)$, all'opposto di coloro che lo conservano nel mobile, che tendono a essere decisamente più attenti a seguirne le indicazioni $(R=0.34$; $\mathrm{p}<0.05)$.

I pazienti che dichiarano di conservare il farmaco "dove capita", mostrando dunque una scarsa attenzione nella conservazione, risultano anche poco soddisfatti nel servizio di ritiro del farmaco presso la propria Asl $(R=-0.55$; $p<0.05)$ e, inoltre, non ritengono importante che il farmacista tenga in considerazione gli aspetti più prettamente attinenti alla terapia all'atto della dispensazione $(R=-0.28 ; p<0.05)$. II dato risulta di assoluto rilievo poiché la $\mathrm{MC}$ sembra chiaramente "circoscrivere" una categoria di pazienti poco attenti agli aspetti più prettamente attinenti alle proprie cura e terapia.

La MC mostra chiaramente come coloro che si somministrano il farmaco, senza delegare "terzi", mantengono questa linea di condotta in maniera spesso ortodossa, ovvero delegano assai raramente la somministrazione del farmaco $(R=-0.66 ; p<0.05)$. Molto interessante rilevare che questi pazienti sono molto attenti anche nei confronti del farmaco stesso, dichiarando infatti di leggere attentamente il foglietto illustrativo $(R=0.33 ; p<0.05)$.

I pazienti che dichiarano di dimenticarsi di assumere la terapia sono spesso anche propensi a interromperla quando si "sentono meglio" $(R=0.33 ; p<0.05)$. Nei pazienti in cui si presentano effetti collaterali legati alla terapia vi sono spesso numerosi casi di abbandono della stessa ( $R=0.49 ; \mathrm{p}<0.05)$; questi tendono però, prima dell'interruzione, a chiedere informazioni al farmacista $(R=0.55 ; p<0.05)$. Coloro che si sentono informati dal farmacista mostrano anche una discreta soddisfazione in relazione alla competenza $(R=0.34 ; p<0.05)$ e alla disponibilità $(R=0.30 ; p<0.05)$ del personale addetto alla consegna; inoltre, gli stessi si ritengono fortemente soddisfatti dell'aiuto ricevuto dal farmacista in caso di necessità $(R=0.74 ; p<0.05)$, dichiarando inoltre di rilevare come, benché il farmacista mostri già una certa attenzione al proprio stato di salute $(R=0.36 ; p<0.05)$, gradirebbero una maggiore attenzione in merito a questo $(R=0.34 ; p<0.05)$ come ad altri aspetti quali lo stato psicologico $(R=0.32 ; p<0.05)$ e quello terapeutico $(R=0.34 ; p<0.05)$.

I pazienti che dichiarano di essere particolarmente soddisfatti del fatto di poter ritirare il farmaco presso la propria Asl risultano anche particolarmente soddisfatti della competenza e della disponibilità $(R=0.52 ; p<0.05)$ mostrate dal personale addetto alla consegna $(R=0.34 ; p<0.05)$; inoltre, rilevano spesso come il farmacista, non solo sia in grado di aiutarli quando si trovano in difficoltà $(R=0.36$; $p<0.05)$ ma come dimostri anche una certa sensibilità sugli aspetti più prettamente terapeutici $(R=0.31 ; p<0.05)$. Appare dunque evidente come il rapporto diretto e personale che si instaura all'atto del "ritiro del farmaco" possa portare a una maggiore soddisfazione del paziente.

Il paziente che dichiara che il farmacista è in grado di aiutarlo quando si trova in difficoltà pare anche fortemente apprezzare il fatto che lo stesso dia particolare importanza agli aspetti connessi alla salute $(R=0.44 ; p<0.05)$ e a quelli psicologici $(R=$ 0.32 ; $p<0.05)$; la loro "sensibilità" su tali aspetti è tale che, alla domanda su quali aspetti il farmacista dovrebbe tenere maggiormente in considerazione, questi rispondono ancora una volta rimarcando esattamente gli stessi identici aspetti.

Per quanto attiene alle correlazioni tra aspetti a cui (prospettiva a) il farmacista pare mostrare maggiore attenzione secondo i pazienti e (prospettiva b) gli aspetti a cui il paziente vorrebbe che il farmacista prestasse maggiore attenzione, vi sono numerose correlazioni che sembrano mettere in evidenza quanto segue. Gli aspetti legati a salute, terapia e psicologia vanno di pari passo per entrambe le prospettive; vale a dire che sono esattamente gli stessi aspetti a cui sembra dare maggiore attenzione il farmacista (prospettiva a) e a cui lo stesso paziente sembra prestare maggiore attenzione (prospettiva b). Per quanto attiene agli aspetti legati alla prescrizione, sembrerebbe che l'attenzione che il farmacista pone alla correttezza della prescrizione non venga notata dal paziente e che allo stesso tempo quest'ultimo non mostri un particolare interesse nel voler ricevere informazioni dettagliate su questo aspetto.

L'analisi dei fattori principali, meglio nota come PFA (prin- 
TABELLA I - Matrice di correlazione di pearson relativa ai parametri indagati $(\mathrm{N}=60)$

\begin{tabular}{|c|c|c|c|c|c|c|c|c|c|c|c|c|c|c|c|}
\hline & AG1 & AG2 & AG3 & AG4 & AG5 & AG6 & AG7 & CF1 & CF3 & CF4 & GFAT1 & GFAT2 & GFAT3 & GFAT4 & GFAT5 \\
\hline AG1 & 1.00 & 0.04 & 0.39 & -0.28 & 0.07 & -0.37 & 0.33 & -0.0 & 0.03 & 0.09 & -0.34 & 0.36 & -0.02 & -0.06 & -0.10 \\
\hline AG2 & & 1.00 & -0.20 & -0.00 & -0.08 & 0.00 & 0.10 & 0.1 & 0.10 & 0.08 & -0.06 & 0.04 & 0.00 & -0.19 & 0.03 \\
\hline AG3 & & & 1.00 & 0.05 & 0.03 & -0.27 & 0.40 & -0.2 & -0.06 & 0.12 & 0.02 & 0.26 & 0.07 & -0.08 & -0.21 \\
\hline AG4 & & & & 1.00 & -0.08 & 0.26 & -0.22 & -0.1 & 0.08 & 0.15 & 0.12 & 0.12 & 0.00 & 0.03 & -0.02 \\
\hline AG5 & & & & & 1.00 & -0.02 & 0.04 & -0.1 & 0.01 & -0.05 & 0.08 & -0.06 & 0.03 & 0.03 & 0.16 \\
\hline AG6 & & & & & & 1.00 & -0.75 & -0.0 & -0.09 & -0.14 & 0.46 & -0.45 & 0.15 & 0.02 & -0.04 \\
\hline AG7 & & & & & & & 1.00 & -0.0 & -0.02 & 0.23 & -0.22 & 0.33 & -0.13 & 0.03 & -0.12 \\
\hline CF1 & & & & & & & & -0.1 & -0.90 & -0.12 & 0.11 & -0.02 & -0.23 & -0.00 & -0.16 \\
\hline CF2 & & & & & & & & 1.0 & -0.18 & -0.02 & -0.35 & -0.03 & -0.35 & 0.18 & -0.06 \\
\hline CF3 & & & & & & & & & 1.00 & -0.13 & 0.01 & 0.05 & 0.34 & -0.08 & 0.19 \\
\hline CF4 & & & & & & & & & & 1.00 & 0.05 & -0.05 & 0.05 & 0.04 & -0.04 \\
\hline GFAT1 & & & & & & & & & & & 1.00 & -0.66 & 0.33 & -0.06 & 0.12 \\
\hline GFAT2 & & & & & & & & & & & & 1.00 & -0.12 & -0.11 & -0.12 \\
\hline GFAT3 & & & & & & & & & & & & & 1.00 & -0.23 & -0.06 \\
\hline GFAT4 & & & & & & & & & & & & & & 1.00 & 0.33 \\
\hline GFAT5 & & & & & & & & & & & & & & & 1.00 \\
\hline & GFAT6 & GFAT7 & GFAT8 & RPF1 & RPF2 & RPF3 & RPF4 & RPF5 & RPF6 & RPF7 & RPF8 & RPF9 & RPF10 & RPF11 & RPF12 \\
\hline AG1 & -0.14 & -0.19 & 0.04 & -0.12 & 0.12 & -0.04 & 0.18 & -0.09 & -0.02 & -0.03 & 0.08 & 0.08 & 0.02 & 0.05 & -0.07 \\
\hline AG2 & 0.13 & 0.07 & 0.08 & -0.16 & -0.17 & -0.09 & -0.09 & -0.31 & -0.11 & -0.03 & -0.02 & -0.18 & 0.01 & -0.24 & -0.13 \\
\hline AG3 & 0.00 & 0.02 & 0.12 & -0.11 & -0.00 & 0.01 & 0.15 & -0.21 & -0.06 & 0.03 & -0.07 & -0.00 & 0.01 & 0.10 & -0.07 \\
\hline AG4 & 0.17 & -0.10 & -0.01 & 0.14 & -0.09 & -0.02 & 0.02 & 0.21 & -0.22 & 0.02 & -0.27 & -0.05 & -0.09 & -0.03 & 0.01 \\
\hline AG5 & -0.01 & 0.12 & -0.08 & -0.17 & -0.16 & -0.20 & -0.16 & -0.08 & 0.15 & -0.07 & 0.20 & 0.15 & 0.17 & 0.13 & 0.16 \\
\hline AG6 & 0.02 & -0.11 & 0.11 & 0.06 & 0.09 & -0.06 & -0.08 & 0.05 & -0.11 & -0.16 & -0.16 & -0.19 & -0.00 & -0.19 & -0.02 \\
\hline AG7 & -0.02 & 0.07 & -0.10 & -0.07 & -0.13 & 0.08 & 0.10 & -0.13 & 0.09 & 0.07 & 0.07 & 0.08 & -0.13 & 0.06 & -0.02 \\
\hline CF1 & -0.10 & -0.06 & -0.12 & -0.03 & 0.03 & -0.09 & -0.01 & -0.01 & -0.10 & 0.03 & -0.09 & 0.14 & 0.08 & 0.14 & 0.15 \\
\hline CF2 & -0.00 & -0.10 & -0.02 & 0.01 & -0.03 & -0.05 & -0.02 & -0.01 & 0.11 & 0.05 & 0.15 & 0.06 & 0.10 & 0.06 & 0.00 \\
\hline CF3 & 0.08 & 0.11 & 0.13 & 0.05 & 0.12 & 0.12 & 0.07 & 0.04 & 0.09 & -0.06 & 0.06 & -0.11 & -0.08 & -0.12 & -0.08 \\
\hline CF4 & 0.08 & -0.07 & -0.02 & -0.09 & -0.55 & -0.04 & -0.19 & -0.10 & -0.12 & 0.03 & -0.09 & -0.20 & -0.13 & -0.18 & -0.28 \\
\hline GFAT1 & 0.14 & 0.15 & 0.05 & -0.06 & 0.01 & -0.02 & -0.01 & -0.12 & -0.13 & -0.10 & -0.19 & -0.18 & 0.09 & -0.18 & -0.13 \\
\hline GFAT2 & -0.11 & -0.13 & -0.05 & -0.05 & 0.05 & 0.03 & -0.01 & 0.15 & -0.08 & 0.10 & -0.00 & 0.11 & -0.16 & 0.15 & 0.07 \\
\hline GFAT3 & 0.04 & -0.12 & 0.05 & -0.16 & -0.04 & -0.06 & -0.06 & -0.16 & -0.03 & -0.10 & -0.03 & -0.10 & -0.05 & -0.07 & 0.06 \\
\hline GFAT4 & -0.09 & -0.01 & -0.13 & -0.12 & -0.13 & -0.16 & 0.06 & 0.04 & -0.09 & 0.11 & -0.16 & -0.08 & 0.07 & -0.08 & -0.07 \\
\hline GFAT5 & 0.10 & 0.13 & -0.04 & -0.20 & 0.03 & -0.08 & -0.03 & -0.05 & -0.09 & 0.08 & -0.13 & -0.10 & 0.16 & -0.08 & -0.13 \\
\hline GFAT6 & 1.00 & 0.49 & 0.55 & 0.23 & -0.07 & -0.10 & 0.06 & 0.08 & -0.06 & -0.15 & -0.18 & 0.10 & -0.12 & 0.07 & 0.11 \\
\hline GFAT7 & & 1.00 & -0.07 & 0.00 & -0.04 & -0.05 & 0.09 & -0.04 & 0.15 & -0.21 & 0.06 & 0.13 & -0.03 & 0.09 & 0.07 \\
\hline GFAT8 & & & 1.00 & 0.19 & -0.02 & -0.04 & -0.01 & -0.10 & -0.12 & 0.03 & -0.09 & -0.00 & 0.07 & -0.00 & 0.09 \\
\hline RPF1 & & & & 1.00 & 0.23 & 0.34 & 0.30 & 0.74 & 0.36 & 0.13 & 0.25 & 0.34 & 0.02 & 0.32 & 0.34 \\
\hline RPF2 & & & & & 1.00 & 0.52 & 0.52 & 0.36 & 0.31 & -0.03 & 0.21 & 0.23 & 0.21 & 0.24 & 0.14 \\
\hline RPF3 & & & & & & 1.00 & 0.40 & 0.36 & 0.44 & 0.07 & 0.34 & 0.38 & 0.17 & 0.36 & 0.17 \\
\hline RPF4 & & & & & & & 1.00 & 0.26 & 0.28 & 0.23 & 0.19 & 0.09 & 0.13 & 0.15 & 0.08 \\
\hline RPF5 & & & & & & & & 1.00 & 0.44 & 0.12 & 0.32 & 0.33 & -0.03 & 0.28 & 0.25 \\
\hline RPF6 & & & & & & & & & 1.00 & 0.12 & 0.87 & 0.47 & 0.17 & 0.44 & 0.43 \\
\hline RPF7 & & & & & & & & & & 1.00 & 0.07 & 0.05 & 0.32 & 0.10 & 0.11 \\
\hline RPF8 & & & & & & & & & & & 1.00 & 0.37 & 0.29 & 0.36 & 0.35 \\
\hline RPF9 & & & & & & & & & & & & 1.00 & 0.12 & 0.93 & 0.76 \\
\hline RPF10 & & & & & & & & & & & & & 1.00 & 0.22 & 0.25 \\
\hline RPF11 & & & & & & & & & & & & & & 1.00 & 0.77 \\
\hline RPF12 & & & & & & & & & & & & & & & 1.00 \\
\hline
\end{tabular}

Per comodità espositiva viene riportata suddivisa in due parti ma in realtà, essendo stata creata incrociando lo stesso numero di fattori per colonna e riga, si tratta di una matrice perfettamente quadrata; in neretto per $p<0.05$.

Le lettere riportate nella Tabella indicano (in ordine di apparizione): $\mathrm{AG1}$ = Età; $\mathrm{AG2}$ = Sesso; $\mathrm{AG} 3$ = status famiglia; $\mathrm{AG} 4$ = titolo studio; $\mathrm{AG5}$ = anni trattamento; AG6 = è lei che acquista i farmaci; AG7 = chi li acquista; CF1 = dove conserva i farmaci: cassetto; CF2 = dove conserva i farmaci: portapillole; CF3 = dove conserva i farmaci: mobile/frigo; CF4 = dove conserva i farmaci: dove capita; GFAT1 = è lei che si somministra i farmaci; GFAT2 = chi le dà i farmaci; GFAT3 = segue le istruzioni; GFAT4 = si dimentica l'assunzione; GFAT5 = se sta bene interrompe la terapia; GFAT6 = effetti collaterali; GFAT7 = diverso da "Mai", ha interrotto la terapia; GFAT8 = prima di interrompere ha chiesto al farmacista; RPF1 = informato dal farmacista; $\mathbf{R P F 2}$ = soddisfazione ritiro presso la sua Asl; RPF3 = soddisfazione competenza del personale addetto alla consegna; RPF4 = soddisfazione disponibilità del personale addetto alla consegna; RPF5 = farmacista l'aiuta se si trova in difficoltà; $\mathbf{R P F 6}$ = aspetti: salute; RPF7 = aspetti: ricetta; RPF8 = aspetti: psicologia; $\mathbf{R P F 9}$ = considerazione: salute; $\mathbf{R P F 1 0}$ = considerazione: ricetta; $\mathbf{R P F 1 1 ~ = ~}$ considerazione: psicologia; RPF12 = considerazione: terapia. 
cipal factor analysis) estrae diversi fattori, con autovalore $>1$ dunque statisticamente significativi (Tab. II); tra questi però il primo (F1) è quello indubbiamente di maggiore importanza poiché è in grado di spiegare oltre il 15\% della varianza totale. Questo fattore estrae i tre aspetti (salute, psicologia e terapia) a cui il farmacista dovrebbe prestare maggiore attenzione secondo i pazienti. Questo fattore ci conferma che, come riportato in precedenza per la MC, gli aspetti legati a salute, terapia e psicologia hanno la stessa rilevanza dal punto di vista del paziente. In particolare sono gli stessi tre aspetti che il paziente vorrebbe fossero presi ugualmente in considerazione dal farmacista.

A seguito dell'elaborazione dei risultati dell'analisi qualitativa è emerso come la medicina narrativa si sia rivelata utile per i pazienti, che hanno avuto la possibilità di ridefinire e rivalutare la propria storia, mostrandosi disponibili al dialogo con il farmacista ospedaliero e rispondendo in maniera esaustiva alle domande proposte durante l'intervista strutturata.

Un aspetto che il lavoro ha evidenziato è che, per quanto concerne la maggior parte dei pazienti, esistono numerose differenze tra il farmacista privato e il farmacista ospedaliero; quest'ultimo viene definito "troppo distaccato", "pignolo", "interessato solo ed esclusivamente alla correttezza della ricetta". Alcune testimonianze affermano il diritto, molto spesso negato, di prendere parola e di proporre un altro punto di vista; è interessante pertanto analizzare la scelta di alcuni pazienti di rivolgersi e affidarsi al solo farmacista privato, con il quale sostengono di avere un rapporto molto più confidenziale. Nonostante questo i pazienti sono consapevoli dell'elevata utenza che la farmacia ospedaliera si trova a gestire e del fatto che i farmaci vengono ritirati una sola volta al mese.

Dalle interviste emergono chiari segnali indirizzati a un rapporto più umano con il farmacista ospedaliero, affermando che è ciò che manca; la maggioranza dei pazienti dializzati ritiene che il farmacista sarebbe una figura molto importante per un reparto, che dovrebbe conoscere i pazienti uno per uno e che potrebbe contribuire efficacemente alla riduzione del problema dell'aderenza alla terapia.

La parola, per molti pazienti, in ambito sanitario non è solo semplice veicolo di informazione, ma può diventare mezzo di cura; è, dunque, necessario che i pazienti vengano posti nella condizione di poter raccontare, scrivere e descrivere. II farmacista deve, a parer loro, essere capace di evocare e guidare la narrazione poiché attraverso questa è possibile instaurare un rapporto di confidenza e un aumento dell'empatia; non solo, emerge la richiesta da parte dei pazienti di una maggiore attenzione alle loro storie personali e alla conoscenza della loro esperienza di malattia.

Sono pazienti che associano e vivono il proprio reparto come una seconda casa ed è opportuno che sia presente il farmacista ospedaliero che dia sostegno concreto e morale ricreando in tal modo un ambiente familiare e che ancora svolga il ruolo di educatore sanitario, responsabile di una corretta informazione sull'utilizzo dei farmaci.

Per molti di loro, il sostegno di un'equipe sanitaria è di fon-
TABELLA II - Pesi fattoriali ( $\mathrm{N}=95)$; metodo di estrazione: principal factor analysis (PFA); metodo di rotazione: varimax. in neretto valori $>0.7$

\begin{tabular}{|c|c|}
\hline PFA & Fattore 1 \\
\hline AG1 & -0.067813 \\
\hline AG2 & -0.159196 \\
\hline AG3 & -0.045596 \\
\hline AG4 & 0.079900 \\
\hline AG5 & 0.319784 \\
\hline AG6 & -0.128235 \\
\hline AG7 & -0.015196 \\
\hline CF1 & 0.130400 \\
\hline CF2 & -0.024315 \\
\hline CF3 & -0.065781 \\
\hline CF4 & -0.217295 \\
\hline GFAT1 & -0.220628 \\
\hline GFAT2 & 0.213523 \\
\hline GFAT3 & 0.021922 \\
\hline GFAT4 & -0.170618 \\
\hline GFAT5 & -0.107339 \\
\hline GFAT6 & 0.118123 \\
\hline GFAT7 & 0.108379 \\
\hline GFAT8 & 0.026405 \\
\hline RPF1 & 0.329425 \\
\hline RPF2 & 0.070458 \\
\hline RPF3 & 0.193642 \\
\hline RPF4 & -0.083442 \\
\hline RPF5 & 0.325263 \\
\hline RPF6 & 0.465959 \\
\hline RPF7 & 0.080448 \\
\hline RPF8 & 0.414542 \\
\hline RPF9 & 0.878661 \\
\hline RPF10 & 0.192295 \\
\hline RPF11 & 0.876830 \\
\hline RPF12 & 0.880742 \\
\hline
\end{tabular}

Le lettere riportate nella Tabella indicano (in ordine di apparizione): $A G 1=$ Età; $A G 2$ = Sesso; $A G 3$ = status famiglia; $A G 4$ = titolo studio; $A G 5$ = anni trattamento; $A G 6$ = è lei che acquista i farmaci; $A G 7$ = chi li acquista; $C F 1=$ dove conserva i farmaci: cassetto; CF2 = dove conserva i farmaci: portapillole; CF3 = dove conserva i farmaci: mobile/frigo; CF4 = dove conserva i farmaci: dove capita; GFAT1 = è lei che si somministra i farmaci; GFAT2 = chi le dà i farmaci; GFAT3 = segue le istruzioni; GFAT4 = si dimentica assunzione; GFAT5 = se sta bene interrompe la terapia; GFAT6 = effetti collaterali; GFAT7 = diverso da "Mai", ha interrotto la terapia; GFAT8 = prima di interrompere ha chiesto al farmacista; RPF1 = informato dal farmacista; RPF2 = soddisfazione ritiro presso la sua Asl; RPF3 = soddisfazione competenza del personale addetto alla consegna; RPF4 = soddisfazione disponibilità del personale addetto alla consegna; $\mathbf{R P F 5}$ = farmacista l'aiuta se si trova in difficoltà; $\mathbf{R P F 6}$ = aspetti: salute; RPF7 = aspetti: ricetta; $\mathbf{R P F 8}$ = aspetti: psicologia; $\mathbf{R P F 9}=$ considerazione: salute; $\mathbf{R P F 1 0}$ = considerazione: ricetta; RPF11 = considerazione: psicologia; RPF12 = considerazione: terapia. 
damentale importanza; il contatto con gli operatori sanitari, che collaborano tra loro, spesso permetterebbe una diagnosi e un accesso alle cure adeguate più veloce e tempestivo.

Al contempo una minoranza non mostra interesse verso la proposta di dialogare con il farmacista e ritiene che il medico sia più che sufficiente in reparto e che le informazioni da lui fornite siano esaustive.

\section{Discussione}

Il lavoro svolto evidenzia che il rapporto paziente-farmacista esiste ma risulta essere poco consolidato, come conferma l'elaborazione statistica, che inoltre sottolinea la necessità per i pazienti che il farmacista sia maggiormente disponibile all'ascolto e quindi acquisisca competenze narrative necessarie a sviluppare un modello di pratica clinica efficace e umana, volto al miglioramento della qualità delle cure e all'appropriatezza dei percorsi assistenziali; la metodica narrativa ha permesso di comprendere la storia di vita dei malati nella loro completezza, le loro esigenze e le loro necessità.

Si evince l'esigenza di intervenire rafforzando l'identità del farmacista ospedaliero come membro dell'equipe sanitaria che prende in carico il paziente all'interno dell'U.O. svolgendo un ruolo di guida, formazione e informazione per la corretta gestione della terapia.

La ricerca ipotizza possibili percorsi di sviluppo: la dispensazione del farmaco potrebbe diventare il momento in cui si consolida l'alleanza terapeutica paziente-farmacista contribuendo, attraverso strategie innovative quali counseling farmacologico e medicina narrativa, a una maggiore aderenza terapeutica con il risultato di rendere il paziente consapevole e partecipe per ottenere un uso responsabile dei farmaci.

\section{Ringraziamenti}

Si ringraziano per i contributi forniti alla discussione e integrati nel testo: Dr. F. Murino (Dirigente Farmacista Nuoro), Dr. G.B. Pintore (Direttore Servizio Farmacia Ospedaliera Nuoro), Dr. G. Capra (Ricercatore Università degli Studi di Sassari).

\section{Disclosures}

Financial support: No financial support was received for this submission.

Conflict of interest: The authors have no conflict of interest.

\section{Appendice 1}

Questionario

Domande di base

1) Età:

2) Sesso: $M \square F \square$

3) Luogo di residenza:

4) Luogo di nascita (se diverso):

5) Status di famiglia (celibe/nubile, sposato/a, separato/a, divorziato/a, vedovo/a, in coppia stabile)

6) Titolo di studio:

7) Attualmente lavora? Sì No (se Sì inserire mestiere) Mestiere:

Professione (se differente):

8) Che tipo di trattamento dialitico segue?

- Emodialisi

- Peritoneale

9) Da quanto tempo è in dialisi? Mese e anno

Domande relative all'acquisto dei farmaci

NB: per le domande che prevedono l'attribuzione di un punteggio seguire la seguente scala o la scala descritta nella domanda:

Mai = 0

Raramente $=1$

Solitamente $\mathbf{=} \mathbf{2}$

Prevalentemente $=\mathbf{3}$

Sempre $=4$

10) Solitamente è lei che si occupa di acquistare i farmaci?

- Mai

- Raramente

- Solitamente

- Prevalentemente

- Sempre

11) Se risponde diversamente da "Sempre", chi in queste occasioni se ne occupa?

- Moglie

- Figlio

- Badante

- Altro:

Domande sulla gestione del farmaco a domicilio (attribuire un valore da 0 a 4 )

12) Dove conserva i farmaci a casa?

Nel cassetto dei farmaci?

Nel portapillole?

Sopraunmobile/nelfrigo?

Dove capita? 
13) Solitamente è lei che si occupa dell'assunzione dei suoi farmaci?

- Mai

- Raramente

- Solitamente

- Prevalentemente

- Sempre

14) Se risponde diversamente da "Sempre", chi in queste occasioni se ne occupa?

- Moglie

- Figlio

- Badante

- Altro:

15) Quando assume il farmaco segue le istruzioni date dal medico/farmacista?

- Mai

- Raramente

- Solitamente

- Prevalentemente

- Sempre

16) Si è mai dimenticato di assumere i farmaci?

- Mai

- Raramente

- Solitamente

- Prevalentemente

- Sempre

17) Quando si sente meglio, interrompe la terapia?

- Mai

- Raramente

- Solitamente

- Prevalentemente

- Sempre

18) I farmaci che assume o che ha assunto le hanno mai dato effetti collaterali lievi?

- Mai

- Raramente

- Solitamente

- Prevalentemente

- Sempre

19) Se risponde diversamente da "Mai", ha interrotto la terapia?

- Mai

- Raramente

- Solitamente

- Prevalentemente

- Sempre

20) Prima di interrompere il farmaco si è rivolto al farmacista?

- Mai

- Raramente

- Solitamente

- Prevalentemente

- Sempre

\section{Domande sul rapporto con il farmacista}

21) Si sente informato riguardo al suo trattamento dal farmacista?

- Assolutamente no

- Poco

- Sufficientemente

- Abbastanza

- Assolutamente sì

22) È soddisfatto di poter ritirare il farmaco presso la sua Asl?

- Assolutamente no

- Poco

- Sufficientemente

- Abbastanza

- Assolutamente sì

23) È soddisfatto del grado di competenza del personale addetto alla consegna del farmaco?

- Assolutamente no

- Poco

- Sufficientemente

- Abbastanza

- Assolutamente sì

24) È soddisfatto della disponibilità del personale addetto alla consegna del farmaco?

- Assolutamente no

- Poco

- Sufficientemente

- Abbastanza

- Assolutamente sì

25) Il farmacista aiuta il paziente se si trova in difficoltà con la terapia?

- Mai

- Raramente

- Solitamente

- Prevalentemente

- Sempre

26) Quali sono gli aspetti a cui prestano più attenzione $i$ farmacisti ospedalieri quando ritira il farmaco? (Attribuire un punteggio secondo la seguente scala: $0=$ assolutamente no, 1 = poco, 2 = sufficientemente, 3 = abbastanza, 4 = assolutamente si)

a. Interesse per la mia salute:

b. Interesse per la correttezza della ricetta:

c. Interesse per il mio stato psicologico:

27) Quali sono gli aspetti che le piacerebbe tenessero in maggiore considerazione i farmacisti ospedalieri quando ritira il farmaco? (Attribuire un punteggio secondo la seguente scala: $0=$ assolutamente no, $1=$ poco, 2 = sufficientemente, 3 = abbastanza, 4 = assolutamente si)

a. Interesse per la mia salute:

b. Interesse per la correttezza della ricetta: 
c. Interesse per il mio stato psicologico/la mia storia:

d. Maggiori delucidazioni/informazioni sulla terapia:

\section{Domande su medicina/farmacia narrativa}

28) Quali sono state le sue impressioni durante il primo incontro con un farmacista ospedaliero? Eventuali differenze con il farmacista privato?

29) Ricorda un episodio significativo?

30) Secondo lei sarebbe opportuno inserire un farmacista in reparto? Un maggiore dialogo con il farmacista potrebbe migliorare la sua condizione?

\section{Bibliografia}

1. Cramer JA, Roy A, Burrell A, et al. Medication Compliance and Persistence: Terminology and Definitions. Value Health. 2008;11(1):44-47.

2. Bordin ES. The generalizability of the psychoanalytic concept of the working alliance. Psychotherapy: Theory, Research \& Practice. 1979;16(3):252-260.

3. Sabaté E. World Health Organization. Aderence to Long-Term Therapies: Evidence for Action. 2003. Geneva: WHO.

4. Martin LR, Williams SL, Haskard KB, Dimatteo MR. The challenge of patient adherence. Ther Clin Risk Manag. 2005;1(3):189-199.

5. Raccomandazione $n^{\circ} 17$ per la Riconciliazione della terapia farmacologica. Ministero della salute. D.G. Programmazione sanitaria, 2014.

6. Consensus Conference, "Linee di indirizzo per l'utilizzo della Medicina Narrativa in ambito clinico-assistenziale, per le malattie rare e cronico-degenerative" aula Rossi, Istituto Superiore di Sanità. Roma, 13 giugno 2014.

7. Good BJ. Narrare la medicina: lo sguardo antropologico sul supporto medico-paziente. 1999. Edizioni di Comunità, Torino.

8. Bert G, Quadrino S. Parole di medici, parole di pazienti. Counseling e narrativa in medicina. Editore II pensiero Scientifico, Roma, 2002.

9. Boskovic J, Mestrovic A, Leppée M, Bago M, Sostar Z, Naletilic D. Pharmacist competences and impact of pharmacist intervention on medication adherence: an observational study. Psychiatr Danub. 2016;28(4):420-427.

10. Kaboli PJ, Hoth AB, McClimon BJ, Schnipper JL. Clinical Pharmacists and Inpatient Medical Care: a Systematic Review.
Arch Intern Med. 2006;166(9):955-964.

11. Faggiano ME, Scala D. "Farmacia Narrativa": la narrazione come strumento per riscoprire e ridefinire la professione del farmacista del SSN. Giornale italiano di Farmacia Clinica. 2016;30(4):190-198.

12. Volino LR, Das RP, Mansukhani RP, Cosler LE. Evaluating the Potential Impact of Pharmacist Counseling on Medication Adherence Using a Simulation Activity. Am J Pharm Educ. 2014;78(9):169.

13. Cooney $\mathrm{D}, \mathrm{Moon} \mathrm{H}$, Liu $\mathrm{Y}$, et al. A pharmacist based intervention to improve the care of patients with CKD: a pragmatic, randomized, controlled trial. BMC Nephrol. 2015;16:56.

14. May R. L'arte del counseling, il consiglio, la guida, la supervisione. 1991. Astrolabio-Ubaldini, Roma.

15. StatSoft Inc Development, 2006. Statistica 7.1. StatSoft Italia SrL, Padova.

16. R Core Team, 2012. R: A language and environment for statistical computing. R Foundation for Statistical Computing, Vienna, Austria Disponibile su http://www.R-project.org/. Accessed April 20, 2017.

17. Garcia R, Millan E. Assessment of $\mathrm{Cd}, \mathrm{Pb}$ and $\mathrm{Zn}$ contamination in roadside soils and grasses from Gipuzkoa (Spain). Chemosphere. 1998;37(8):1615-1625.

18. Reimann C, Filzmoser P, Garrett RG. Factor analysis applied to regional geochemical data: Problems and possibilities. Appl Geochem. 2002;17:185-206.

19. http://www.sanita24.ilsole24ore.com/art/medicina-ericerca/2016-03-11/la-malattia-renale-cronica-italia-numerie-costi-104146. php?uuid = ACLp3JmC. Accessed April 202017. 